Check for updates

Cite this: RSC Adv., 2017, 7, 50772

Received 10th August 2017

Accepted 29th September 2017

DOI: $10.1039 / c 7 r a 08855 h$

rsc.li/rsc-advances

\section{Resistant starch attenuates impaired lipid biosynthesis induced by dietary oxidized oil via activation of insulin signaling pathways $\dagger$}

\begin{abstract}
Wenting Shang, (DD a Xu Si, ${ }^{a}$ Padraig Strappe, ${ }^{\mathrm{b}}$ Zhongkai Zhou*ac and Chris Blanchard ${ }^{\mathrm{c}}$
The current study found that deep-frying process led to an increased content of oxidized triacylglycerols in canola oil, 3.5 times higher than that of fresh canola oil (not used for frying). A rat model was then used to study the effect of the consumption of oxidized oil on blood lipid compositions and investigate the mechanism involved in lipids metabolism in the liver of rats with resistant starch (RS) intervention. Studies involving animals revealed that the consumption of deep-fried oil (DO group) significantly reduced the level of both triglycerides and high-density lipoprotein cholesterols $(P<0.05)$ in the serum, indicating that lipid biosynthesis was impaired. However, the supplementation of RS in the DO-containing diet (DO-RS group) attenuated the abovementioned status. The further study found that Insig expression was down-regulated with an increased mRNA expression of PPAR $\alpha$, together with reduced expressions of SREBP1 and downstream lipogenesis-associated genes in the rats of the DO group. In contrast, RS supplementation up-regulated Prkag2 (an AMPK related gene) and Insig-1/2 expressions in the DO-RS group compared to that in the DO group. The activation of the Insig pathway might be one of the key regulators for attenuating the impaired lipid biosynthesis induced by the oxidized fat following RS intervention.
\end{abstract}

\section{Introduction}

Deep-frying is one of the most popular cooking methods worldwide due to its convenience and the unique effect on food flavor and texture, ${ }^{1}$ which leads to a higher contribution of oxidized fat to the total energy intake. The unstable nature of oil components during the deep-frying process results in a number of chemical reactions occurring within the oils that have been used for frying, which leads to the formation of a mixture of chemically distinct lipid peroxidation products. ${ }^{2}$ These reactions influence not only the physicochemical properties of oil ${ }^{3}$ but also its nutritional properties due to the generation of toxic compounds such as peroxides, aldehydes, ketones, free radicals, and trans fatty acids. ${ }^{4}$

It has been reported that foods such as potato chips fried between 150 and $180^{\circ} \mathrm{C}$ absorb 8 to 25 percent of oil, ${ }^{5}$ indicating that oils that have been used for frying is becoming one of the

${ }^{a}$ Key Laboratory of Food Nutrition and Safety, Ministry of Education, School of Food Engineering and Biotechnology, Tianjin University of Science and Technology, Tianjin 300457, China. E-mail: zkzhou@tust.edu.cn; Fax: +86 2260601371; Tel: +86 2260601408

${ }^{b}$ School of Medical and Applied Sciences, Central Queensland University, Rockhampton, Qld 4700, Australia

${ }^{c} A R C$ Industrial Transformation Training Centre for Functional Grains, Charles Sturt University, Wagga Wagga, NSW 2678, Australia

$\dagger$ Electronic supplementary information (ESI) available. See DOI: 10.1039/c7ra08855h major dietary components. An increasing concern is the deleterious effect of deep-oils that have been used for frying on human health, ${ }^{6}$ and previous investigations have indicated that lipid oxidation positively influences the formation of acrylamide. ${ }^{-10}$ Moreover, it is confirmed that some products generated from lipid oxidation pose a chronic threat to health by exerting cytotoxic and genotoxic effects. ${ }^{11}$ Previous research in rats and pigs models has found that the ingestion of oxidized oils provokes various effects such as depletion of antioxidants, increase in lipid peroxidation, ${ }^{12-14}$ impairment of glucose tolerance $^{15}$ and alterations of thyroid hormone homeostasis. ${ }^{16-18}$ In addition, a great number of studies have also revealed that the consumption of oxidized oils can affect lipid metabolism, in particular, lowering the concentration of blood lipids. The literature strongly indicates that the lowering of blood lipids as induced by oxidized oil consumption is not due to the potentially confounding effects of reduced energy intake and food intake, decreased nutrient digestion and absorption, lowing of endogenous antioxidation capacity, and decreased levels of essential fatty acids but rather mediated directly by inducing the alterations in lipid metabolism in the host. ${ }^{19}$ However, current understanding of the molecular mechanisms underlying these alterations in blood lipid metabolism induced by dietary oxidized oil consumption is not completed.

Given that the frying process can result in absorbed oil contributing to almost 25 percent in the corresponding food, ${ }^{5}$ 
a more detailed evaluation of the toxic effects of oxidized oilcontaining diet is needed. Despite previous research on the effects of the pure toxic compounds isolated from deep-oils that have been used for frying on human health, a comprehensive study of an animal model incorporating the effects of direct consumption of oxidized oil-containing diet is necessary to assess its potential toxic properties. To date, the literature is lacking sufficient studies that elucidate these toxic mechanisms and what potential dietary interventions may alleviate this toxicity. More interestingly, among the dietary interventions, the utilization of resistant starch (RS) in the food system is becoming more and more popular due to its benefit to the gut environment via the fermentation of microbiota. ${ }^{20}$ Resistant starch is the fraction of dietary starch that escapes digestion in the small intestine and passes into the large bowel of healthy humans, thereby, contributing to total dietary fibre intake. ${ }^{21}$ Previous studies have indicated that the consumption of RS might help to maintain a healthy body weight through manipulating glycol-metabolism and lipid metabolism, ${ }^{22-24}$ reducing the risk of intestinal diseases and facilitating beneficial intestinal probiotic growth and mineral absorption. ${ }^{25,26}$ Our study also demonstrated that RS induces protective anti-oxidant responses in high-fat diet fed rats. ${ }^{27}$ Recently, Kieffer et al. $(2016)^{28}$ have shown that an RS supplemented high-fat diet had a profound effect on liver metabolism and gene expression profiles, which were primarily associated with a specific change in gut bacteria. Thus, this study employs the combination of molecular and histological techniques to understand the different impacts of deep-fried oil consumption on lipid metabolism in the rat liver with or without the incorporation of RS into the diet.

\section{Materials and methods}

\subsection{Materials}

Resistant starch (RS), from high amylose maize starch (Himaize $^{\mathrm{TM}}$ ), was obtained from Ingredion Company (NSW, Australia). Other chemicals were of reagent grade and used as received. Fresh canola oil (FO) (i.e. canola oil not yet used for frying) was purchased from a local supermarket.

Oil containing high level of oxidized products was prepared by deep-frying process as described previously. ${ }^{29}$ In brief, the fresh canola oil was heated at $190 \pm 5{ }^{\circ} \mathrm{C}$ for 5 intermittent days ( $7 \mathrm{~h}$ each day) for a total of $35 \mathrm{~h}$. Fresh canola oil $(7 \mathrm{~L})$ was poured into an iron saucepan with a bore of $45 \mathrm{~cm}$ and a depth of $20 \mathrm{~cm}$, and $100 \mathrm{~g}$ of chicken nuggets, potato chips, bread pieces, or fish were fried for 4 or $2 \mathrm{~min}$ in succession for $30 \mathrm{~min}$ each without replenishing.

\subsection{Lipidomics analysis of oil composition}

Briefly, $20 \mu \mathrm{L}$ of each oil sample was dissolved in $1 \mathrm{~mL}$ of chloroform : methanol $(1: 1)(\mathrm{v} / \mathrm{v})$ in glass vials. The samples were then spiked with appropriate concentrations of deuterated diacylglycerols (DAG) and triacylglycerol (TAG) species, including DAG16 : 0/16 : 0-d5, DAG18:1/18 : 1-d5 (Avanti Polar Lipids, Alabaster, AL, USA), d5-TAG (16:0) 3, TAG (14:0) 3-d5,
TAG (15: 0) 3-d29 (CDN isotopes) for analysis on a Shimadzu Exion UPLC coupled with SCIEX QTRAP 6500 Plus. Neutral lipids (DAGs and TAGs) were analyzed using a modified version of reverse phase HPLC/ESI/MS/MS based on neutral loss scanning as described previously. ${ }^{30}$ Separation of lipids aforementioned was carried out on a Phenomenex Kinetex $2.6 \mu-\mathrm{C} 18$ column (i.d. $4.6 \times 100 \mathrm{~mm}$ ) using an isocratic mobile phase chloroform : methanol : $0.1 \quad \mathrm{M} \quad$ ammonium acetate $(100: 100: 4)$ at a flow rate of $150 \mu \mathrm{L} \min ^{-1}$ for $22 \mathrm{~min}$.

\subsection{Animals and diets}

Wistar rats of $295 \pm 10 \mathrm{~g}$ weight, which were obtained from the animal house, Chinese Military Medical Science Academy, were used for the animal model study. After adaptive feeding with the basal diet for one week, 32 rats were randomly divided into four groups. Group 1: basal diet without extra oil consumption (normal control, NC group); Group 2: basal diet plus extra unheated canola oil (FO group); Group 3: basal diet plus deepfried oil (DO group); Group 4: basal diet plus deep-fried oil and RS (DO-RS group). For the FO and DO groups, rats were fed with $1.5 \mathrm{~mL}$ unheated or heated oil, respectively, once daily for 6 consecutive weeks by oral gavage using a feeding needle. Specifically, for the DO-RS group, before oral gavage, deep-fried oil and RS were well mixed following the ratio of $1.5: 2(\mathrm{v} / \mathrm{g})$. Each group consisted of 8 animals housed in plastic cages (4 rats per cage) with free access to food and water. The conditions of humidity $(55 \pm 5 \%)$, light $(12 / 12 \mathrm{~h}$ light/dark cycle) and temperature (at $23{ }^{\circ} \mathrm{C}$ ) were controlled throughout the entire experiment. The main ingredients of the basal diet (standard rodent chow) are shown in Table S1. $\dagger$

\subsection{Analysis of serum lipid profile}

At the end of the six-week experimental period, the rats were fasted overnight and the blood samples were collected from the femoral artery before the animals were sacrificed by cervical dislocation. The serum samples were prepared by centrifugation at $4690 \times g$ for $15 \mathrm{~min}$ and then stored at $-80^{\circ} \mathrm{C}$ prior to biochemical analyses. Total cholesterol (TC), triglyceride (TG), low-density lipoprotein cholesterol (LDL-c), and high-density lipoprotein cholesterol (HDL-c) concentrations were measured according to the instructions of the corresponding kits (Jiancheng Biological Engineering Institute, Nanjing, China). Following the cervical dislocation, the rats were dissected immediately with sterile scissors and their livers were removed, weighed, and immediately frozen in liquid nitrogen and stored at $-80^{\circ} \mathrm{C}$ prior to RNA extraction.

\subsection{Histological analysis}

After dissection of all animals, the aorta was removed and fixed in $10 \%$ neutral formalin for $48 \mathrm{~h}$ and washed in running tap water for $24 \mathrm{~h}$. The tissues were then dehydrated using $30 \%$, $50 \%, 70 \%, 80 \%, 90 \%, 95 \%$, and $100 \%$ ethanol, cleared in two changes of xylene, embedded in paraffin (BMJ-III embedding machine, Changzhou Electronic Instrument Factory, Jiangsu, China), and then cut into $5 \mu \mathrm{m}$ thick sections using a microtome (Leica RM2235; Leica, Heidelberg, Germany). The slides 
were stained using haematoxylin and eosin ( $\mathrm{H} \& \mathrm{E}$ ) for histological examination.

\subsection{Quantitative RT-PCR analysis and digital gene expression tag profiling}

Total RNA was extracted from the liver sample using Trizol Reagent, and Q-RT-PCR analysis was performed. Three replicates of each sample were tested, and the relative transcript quantity was calculated with $18 S$ rRNA gene as an internal control following the method of $2^{-\Delta \Delta C T} 3^{31}$

The mRNA was sheared into short fragments by adding a fragmentation buffer. First-strand cDNA was synthesized using random primers and Superscript II. DNA polymerase I and RNase $\mathrm{H}$ were used to generate second-strand cDNA. Double-stranded cDNA was end-repaired by adding T4 DNA polymerase, Klenow Enzyme and T4 polynucleotide kinase. This was followed by a single 'A' base addition using Klenow 3-5' exopolymerase, and then sequencing adapters were ligated to the fragments using DNA ligase. The cDNA library was created after the cDNA fragments (PE200) were separated by agarose gel electrophoresis and then sequenced on the IlluminaHiseq ${ }^{\mathrm{TM}}$ 2500 platform.

Transcript abundance and differential gene expression were calculated using the program Cufflinks. ${ }^{32}$ The $P$ value threshold was determined by the false discovery rate (FDR) to account for multiple tests of significance. In this study, an FDR threshold $\leq$ 0.01 and a fold change $\geq 2$ were considered as significant differences in gene expression.

\subsection{Ethical statement}

All animal procedures were performed in accordance with the National Institutes of Health's Guide for the Use and Care of Laboratory Animals (NIH Publication No. 85-23, revised 1996), and this study was approved by the Tianjin Institutional Animal Care and Use Committee.

\subsection{Statistical analysis}

The results are given as mean \pm standard deviation (SD) values. The significant difference from the respective control for each experimental test condition was assessed by one-way analysis of variance (ANOVA) and two-tailed Student's $t$ test, and $P<0.05$ was considered statistically significant.

\section{Results}

\subsection{Analysis of DAGs, TAGs and oxidized TAGs species in canola oil before and after deep-frying}

Deep-frying of canola oil resulted in an increase in DAGs although this change was not significant compared to non-fried oil (Fig. 1a). More importantly, this study found that deep-frying led to a significant reduction in the level of TAGs species in canola oil $(P<0.05)$ (Fig. 1a). Considering that the concentration of TAGs species in canola oil is far greater than that of DAGs (1.7524 versus $0.0417 \mathrm{moL}$ lipid per L oil), the changes in the concentration of its oxidized species before and after deepfrying process were also determined and the results are
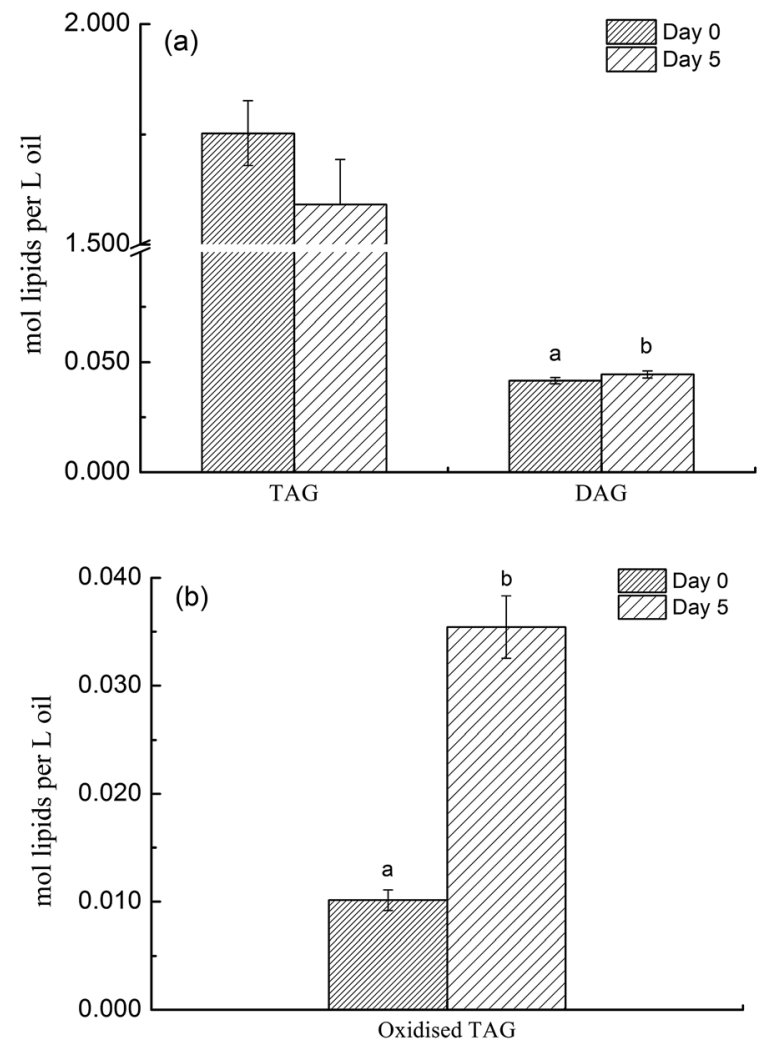

Fig. 1 The difference in the content of DAGs, TAGs and oxidized TAGs species between non- and deep-fried oils (day 0 versus day 5 ). Different superscript lowercase letters indicate significant difference at $P<0.05$ between treatments.

presented in Fig. 1b, showing that deep-frying process led to a 3.5 times increase in the level of oxidized TAGs compared to that in non-fried oil (0.0354 versus 0.0101 moL lipid per L oil).

\subsection{Molecular profile of DAGs, TAGs and oxidized TAGs species in canola oil before and after deep-frying}

The lipidomics data also showed that canola oil consisted a total of 259 TAGs and 15 DAGs species. The molecular profiles of 60 major representative TAGs and all 15 DAGs species are displayed in Fig. 2. It is interesting to note that, except for an increase in the concentration of DAG36:2(18:1), deep-frying did not result in significant changes in the molecular profile of other DAGs species (Fig. 2a), indicating their higher stability during deep-frying compared to that of TAGs. In contrast, the concentration of a number of TAGs species, including TAG54 :4 (18:1), $54: 5$ (18:1), $54: 5$ (18:2), $54: 5$ (18:3), and $54: 6$ $(18: 2)$, which were all unsaturated fatty acids, was found to be significantly reduced due to the deep-frying (Fig. 2b).

Furthermore, lipidomics analysis revealed a total of 58 oxidized TAGs species in canola oil and deep-frying greatly promoted the formation of oxidized TAGs including TAG $54: 3-$ $18: 1 \mathrm{OH}, 54: 4(\mathrm{OH})-18: 2,54: 4-18: 2 \mathrm{OH}$, and $54: 4-18: 1$ $\mathrm{OH}$. The accumulation of the oxidized TAGs species in DO is related to the loss of the corresponding TAGs species in its original state (un-fried sample) during the deep-frying process. 

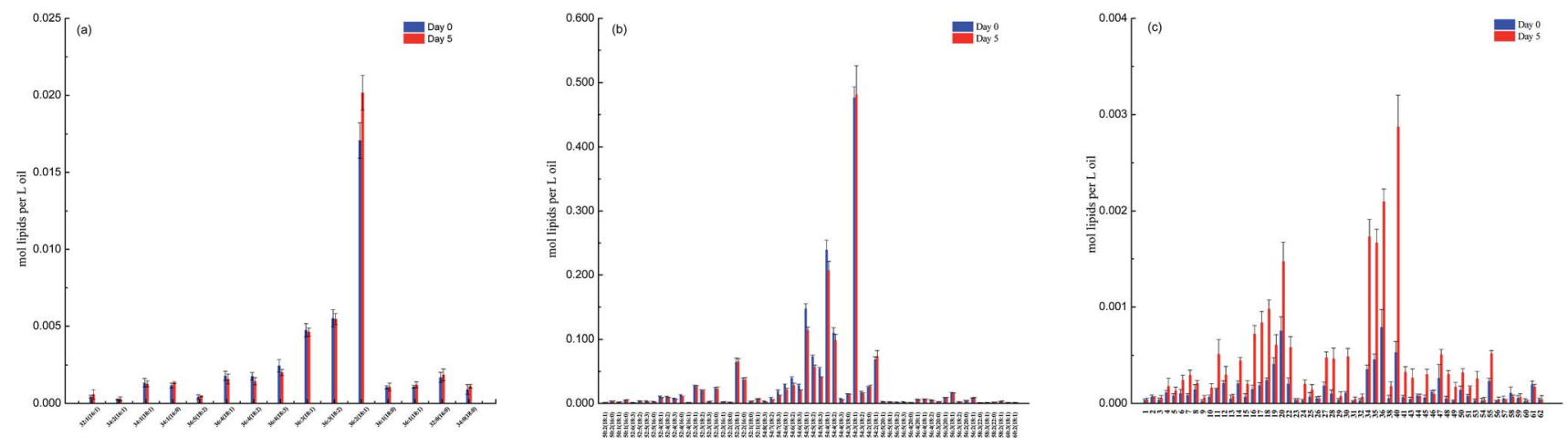

Fig. 2 The difference in the molecular profile of DAGs, TAGs and oxidized TAGs species between non- and deep-fried oils (day 0 versus day 5). (a) DAGs; (b) TAGs; and (c) oxidized TAGs.

Table 1 Levels of TG, TC, LDL-C and HDL-C in the serum of rats ${ }^{a}$

\begin{tabular}{lllll}
\hline Group & TG & TC & LDL-c & HDL-c \\
\hline NC & $2.43 \pm 0.29^{\mathrm{a}}$ & $2.22 \pm 0.09^{\mathrm{a}}$ & $0.62 \pm 0.09^{\mathrm{a}}$ & $0.92 \pm 0.17^{\mathrm{a}}$ \\
FO & $2.19 \pm 0.63^{\mathrm{ab}}$ & $2.21 \pm 0.29^{\mathrm{ab}}$ & $0.63 \pm 0.08^{\mathrm{a}}$ & $0.77 \pm 0.11^{\mathrm{a}}$ \\
DO & $1.52 \pm 0.30^{\mathrm{b}}$ & $1.83 \pm 0.16^{\mathrm{b}}$ & $0.55 \pm 0.08^{\mathrm{a}}$ & $0.45 \pm 0.10^{\mathrm{b}}$ \\
DO-RS & $1.84 \pm 0.67^{\mathrm{ab}}$ & $1.93 \pm 0.19^{\mathrm{ab}}$ & $0.39 \pm 0.04^{\mathrm{b}}$ & $0.89 \pm 0.16^{\mathrm{a}}$
\end{tabular}

${ }^{a}$ The results are expressed as means \pm SD $(n=8$, one-way ANOVA). Different superscript lowercase letters above the same column indicate significant difference $(P<0.05)$. TG: triglyceride; TC: total cholesterol; LDL-c: low-density lipoprotein cholesterol; HDL-c: highdensity lipoprotein cholesterol. NC: normal control; FO: fresh canola oil (unheated oil); DO: deep-fried oil; DO-RS: deep-fried oil plus resistant starch.

\subsection{Blood lipids profile}

The lipid levels in the serum were detected using an automatic biochemical analyzer, and the results are shown in Table 1 . The levels of TG, TC, LDL-c, and HDL-c in the serum of rats in deepfried oil (DO) group were significantly reduced $(P<0.05)$ compared with those in the normal control (NC) group. In contrast, for the animals which received a diet containing both DO and RS (DO-RS group), the status of TG, TC, LDL-c, and HDL-c was returned to a nearly normal level of the NC group $(P<$ 0.05) (Table 1), indicating a positive influence on blood lipid biosynthesis following RS intervention.

\subsection{Liver index}

The liver index of the rats was calculated from the ratio of liver weight to body weight (expressed as a percentage) for each group, and the results are presented in Fig. 3. A diet containing oxidized oil (DO group) significantly reduced the liver index of rats compared to that for the FO group. However, RS intervention (DO-RS group) resulted in a similar liver index to that of the rats in both the FO and NC groups, suggesting an improvement in lipid metabolism by RS dietary supplementation.

\subsection{Blood vessel histology}

Histological analysis of aorta sections was performed for the animals in each group, and the results are presented in Fig. 4. In general, histological abnormalities were not observed in the rats of the NC group (Fig. 4A). However, the internal elastic membrane of aorta sections of the rats in the DO group was found to be compromised (Fig. 4B) and atherosclerotic plaque formation was also observed in $2 / 8$ cases. Furthermore, the narrowing of the aortic lumen to the middle bulge was also seen in $2 / 8$ cases (Fig. $4 \mathrm{C}$ ). In contrast, RS supplement in the oxidized oil-containing diet (i.e. the DO-RS group) improved the above status, in which 7 animals showed histology similar to that of the normal status (Fig. 4D and E) with only 1 case displaying atherosclerotic plaques.

\subsection{Functional annotation and classification of differentially expressed genes}

To comprehensively investigate the liver gene transcriptional response to DO in the diet and the effect of RS intervention, four DGE libraries were constructed from the livers of rats in the NC, FO, DO and DO-RS groups by applying Illumina technology, generating more than 4 gigabases (GB) of transcriptome data from each library, and the read numbers aligned onto the rat reference genome are summarized in Table $\mathrm{S} 2 . \dagger$

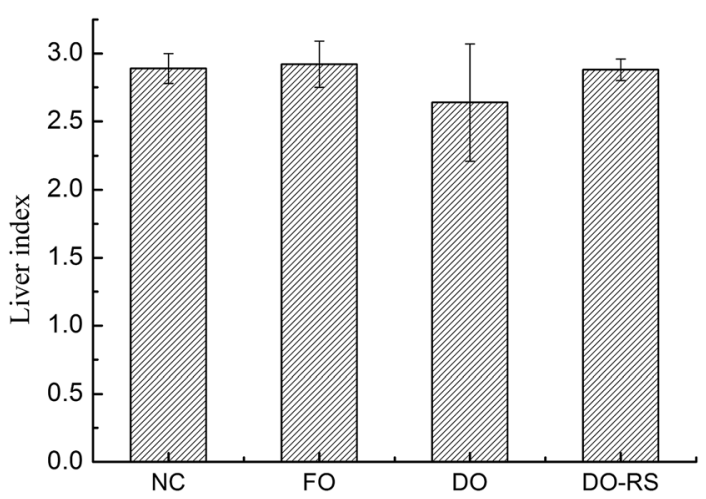

Fig. 3 Effect of the consumption of deep-fried oil either with or without RS intervention on the liver index of rats. The results are expressed as means \pm SD. NC: normal control; FO: fresh canola oil (non-fried oil); DO: deep-fried oil; DO-RS: deep-fried oil plus resistant starch. 

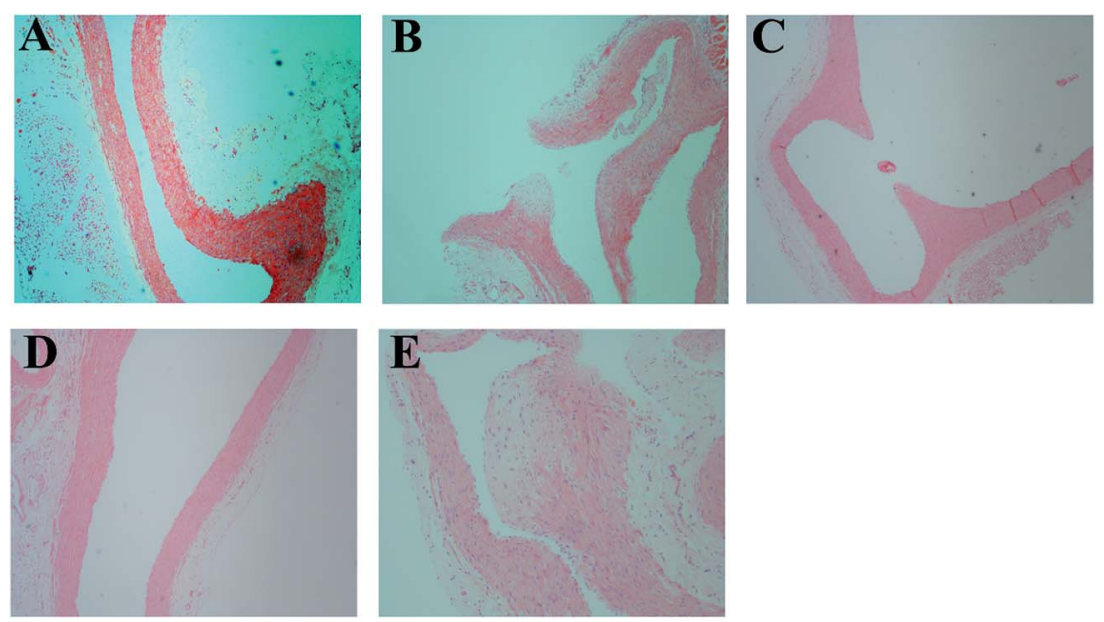

Fig. 4 Histological morphology of the aorta sections of rats in different groups. (A) rats fed with basal diet, (B) and (C) rats fed with basal diet plus deep-fried oil, (D) and (E) rats fed with basal diet plus deep-fried oil and RS.

A total of 429 genes were differentially expressed in the FO group versus the DO group, and 384 genes were differentially expressed in the FO group versus the DO-RS group. The GO enrichment analysis (Fig. S1†) showed that the differentially expressed genes in the FO group versus the DO group and in the DO group versus the DO-RS group were mainly related to biological process, cellular component and molecular function. More importantly, comparing the FO group with the DO group, 47 significant enrichment pathways were mapped. Furthermore, 21 significant enrichment pathways were detected in the DO group versus the DO-RS group and 6 pathways were related to lipid metabolism, including linoleic acid metabolism, AMPK signaling pathway, insulin signaling pathway, arachidonic acid metabolism, glycosphingolipid biosynthesis-ganglio series and glycerophospholipid metabolism (Table 2). These results might indicate that the RS supplementation in the oxidized oilcontaining diet may act as an important regulator of lipids biosynthesis at the molecular level.

\subsection{AMPK signaling pathway}

This study found that the AMPK signaling pathway was significantly enriched compared the DO group with the DO-RS group (Table 2), and further data in the KEGG pathway analysis showed that compared with that in the DO group, Prkag2, an AMPK related gene, was up-regulated in the DO-RS group. In contrast, sterol regulatory element binding transcription factor

Table 2 Significantly enriched functional pathways of gene regulation induced by treatment between the DO group and the DO-RS group ${ }^{a}$

\begin{tabular}{|c|c|c|c|c|}
\hline Pathway name & Pathway class & $P$ value & FDR & Pathway id \\
\hline FoxO signaling pathway & Signal transduction & 0.00025 & 0.041822 & rno04068 \\
\hline Herpes simplex infection & Infectious diseases: viral & 0.00062 & 0.04792 & rno05168 \\
\hline Linoleic acid metabolism & Lipid metabolism & 0.001172 & 0.048917 & rno00591 \\
\hline Circadian rhythm & Environmental adaptation & 0.005486 & 0.142293 & rno04710 \\
\hline AMPK signaling pathway & Signal transduction & 0.00574 & 0.142293 & rno04152 \\
\hline Jak-STAT signaling pathway & Signal transduction & 0.009489 & 0.176079 & rno04630 \\
\hline Arachidonic acid metabolism & Lipid metabolism & 0.013453 & 0.224673 & rno00590 \\
\hline Prolactin signaling pathway & Endocrine system & 0.016238 & 0.235055 & rno04917 \\
\hline Glycosphingolipid biosynthesis-ganglio series & Glycan biosynthesis and metabolism & 0.01689 & 0.235055 & rno00604 \\
\hline Hepatitis C & Infectious diseases: viral & 0.021481 & 0.275947 & rno05160 \\
\hline Type II diabetes mellitus & Endocrine and metabolic diseases & 0.024889 & 0.292024 & rno04930 \\
\hline Inflammatory bowel disease (IBD) & Immune diseases & 0.038924 & 0.342121 & rno05321 \\
\hline Dorso-ventral axis formation & Development & 0.044125 & 0.360661 & rno04320 \\
\hline Steroid hormone biosynthesis & Lipid metabolism & 0.046198 & 0.360661 & rno00140 \\
\hline
\end{tabular}

${ }^{a}$ FO: fresh canola oil (unheated oil); DO: deep-fried oil; DO-RS: deep-fried oil plus resistant starch. 


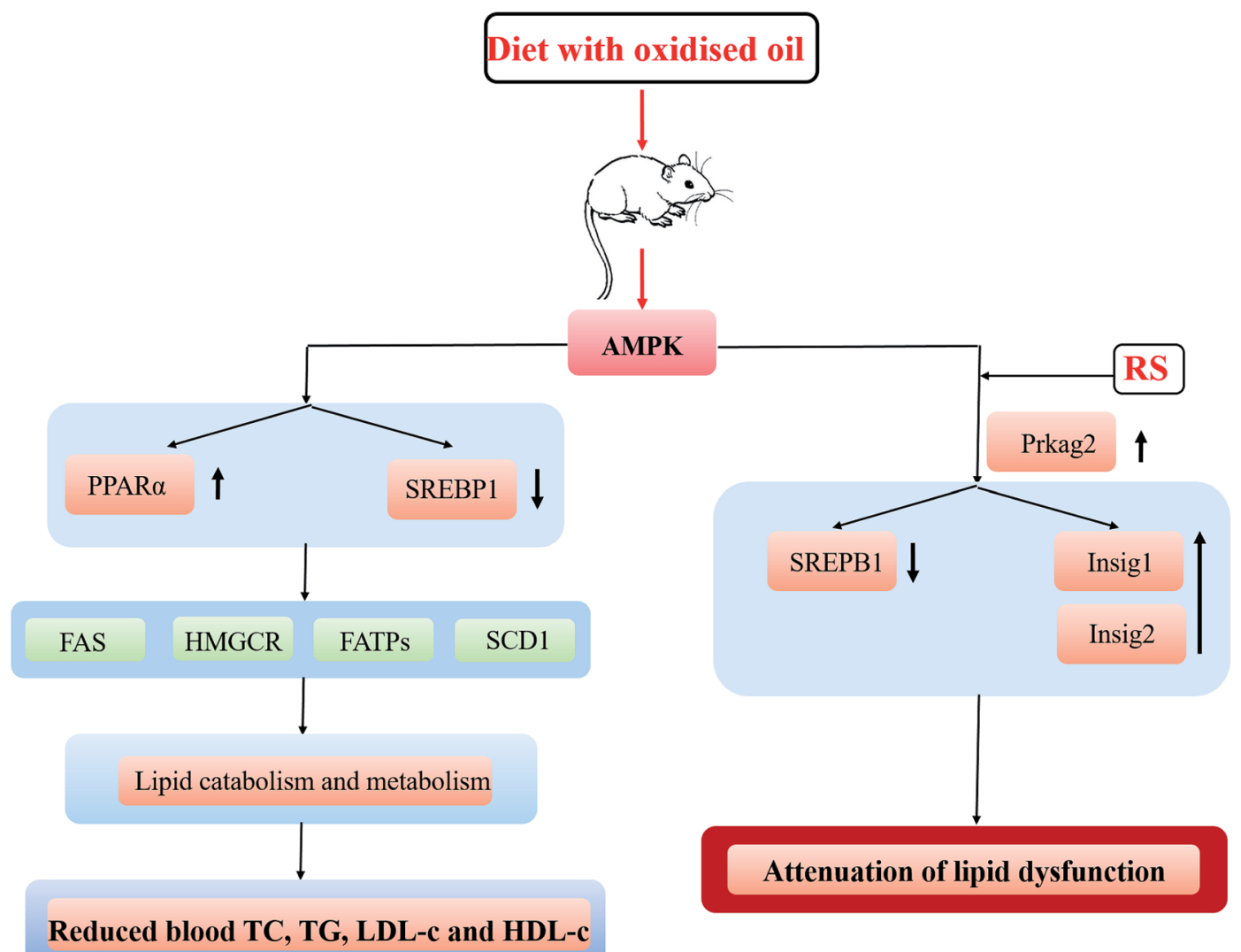

Fig. 5 A proposed mechanism for interpretation of the impact of oxidized oil consumption either with or without RS on lipid metabolism.

1 (SREBF1) and stearoyl CoA desaturase-1 (SCD1) were downregulated in the DO-RS group (Fig. 5A). Furthermore, a lipogenic transcription factor, which is peroxisome proliferator activated receptor $\gamma(P P A R \gamma)$, was found to be activated in the DO group, whilst the expression of lipogenesis-associated genes, such as sterol regulatory element binding proteins (SREBPS), fatty acid synthase (FAS), 3-hydroxy-3-methylglutaryl coenzyme A $(H M G-C O A)$ reductase $(H M G C R)$, fatty acid transport proteins (FATPS), and fatty acid-binding proteins (FABPS) in the digital gene expression (DGE) libraries was noticed to be reduced in the DO group compared to those in the NC group.

\subsection{Insulin signaling pathway}

The insulin signaling pathway was also significantly enriched in the DO group versus the DO-RS group (Table 2). Insulin regulates lipogenesis through the activation of SREBPs, ${ }^{33}$ and the previous report has described that over-expression of Insig-1 in the liver of transgenic mice inhibits SREBP processing and reduces insulin-stimulated lipogenesis. ${ }^{34}$ In this study, compared with that of the rats in the FO group, Insig-1 expression was down-regulated in the rats of the DO group, while the expression of Insig-1 was up-regulated and SREBP1 was downregulated in the rats of the DO-RS group compared with those of the rats in the DO group.

\subsection{Expression of lipid metabolism related genes}

To explore the molecular mechanism of RS in relation to the regulation of lipid metabolism, the mRNA expression levels of genes related to lipid metabolism were measured in the rat liver using a real-time quantitative PCR (Table 3). The data

Table 3 Quantitative RT-PCR analysis of gene expression of lipid metabolism in the livers of rats ${ }^{a}$

\begin{tabular}{|c|c|c|c|c|c|c|c|}
\hline NC & $1.00^{\mathrm{a}}$ & $1.00^{\mathrm{a}}$ & $1.00^{\mathrm{a}}$ & $1.00^{\mathrm{a}}$ & $1.00^{\mathrm{a}}$ & $1.00^{\mathrm{a}}$ & $1.00^{\mathrm{a}}$ \\
\hline FO & $0.89 \pm 0.07^{\mathrm{a}}$ & $0.49 \pm 0.04^{\mathrm{b}}$ & $0.60 \pm 0.02^{\mathrm{a}}$ & $0.61 \pm 0.02^{\mathrm{a}}$ & $0.68 \pm 0.06^{\mathrm{a}}$ & $0.60 \pm 0.03^{\mathrm{a}}$ & $0.58 \pm 0.03^{\mathrm{a}}$ \\
\hline DO-RS & $0.77 \pm 0.06^{\mathrm{a}}$ & $0.62 \pm 0.05^{\mathrm{a}}$ & $0.51 \pm 0.04^{\mathrm{b}}$ & $0.45 \pm 0.02^{\mathrm{b}}$ & $0.44 \pm 0.07^{\mathrm{b}}$ & $0.55 \pm 0.03^{\mathrm{a}}$ & $0.60 \pm 0.04^{\mathrm{a}}$ \\
\hline
\end{tabular}

${ }^{a}$ The results are expressed as mean $\pm \mathrm{SD}$ of triplicate determinations $(n=8)$. Different superscript lowercase letters above the same column indicate significant difference at $P<0.05$ between treatments. NC: normal control; FO: fresh canola oil (unheated oil); DO: deep-fried oil; DORS: deep-fried oil plus resistant starch. 
demonstrated that the mRNA expression of Insig-1 decreased in the rats of the DO group compared with that in the rats of the NC and FO groups, while it was increased after RS intervention compared to that in the rats of the DO group $(P<0.05)$. Moreover, compared to that in the rats of the other groups, downregulation of Insig-2 expression in the rats of the DO group was also noticed although the difference was not significant. As shown in Table 3, RS supplementation in the oxidized oilcontaining diet (DO-RS group) effectively reduced the SREBP-1 mRNA level in the liver of rats compared with that in the rats of the DO group $(P<0.05)$. Fatty acid synthesis gene, FADS1, and triglyceride synthesis-related genes (GPAM and DGAT1) were down-regulated following the oil consumption, and the oxidized oil-containing diet led to the lowest expression level of the above genes $(P<0.05)$. In contrast, RS supplementation in the oxidized oil-containing diet caused a significant change in the expression of these genes (DO versus DO-RS group). These results were consistent with the corresponding data of the compositions of blood lipids in Table 1.

\section{Discussion}

Lipidomic analysis showed that deep-frying led to a significant reduction of TAGs concentration accompanied by a pronounced increase in the level of oxidized TAGs, with a slight increase in the content of DAGs. These results demonstrate the relative stability of different lipid species during the thermal process in which TAGs species are more likely to be degraded and oxidized with the observed changes in the concentrations of TAGs (reduced), DAGs (increased), and oxidized TAGs (increased). This is the first study to highlight this relationship in the context of deep-frying. In particular, unsaturated fatty acids in the TAGs species are highly involved in these two chemical reactions.

Although a number of studies on the impact of dietary oxidized oil consumption on human health have been performed, in this study, we found a paradoxical relationship between the decreased blood TG, TC, LDL-c and HDL-c levels (Table 1) and the liver index (Fig. 3) associated with the consumption of the oxidized oil-containing diet. Moreover, we found that the abdominal (epididymal and perinephrial) adipose tissue weights were also influenced by the oxidized oil consumption although the difference was not significant compared to the other three groups (Table S $3 \dagger$ ), suggesting that the oxidized oil-containing diet impairs lipid biosynthesis and metabolism. Nevertheless, the consumption of oxidized oil is believed to elevate the blood pressure ${ }^{35}$ and promote vascular inflammation. $^{36}$ Our present results showed that the RS supplementation reversed the toxic impact of the oxidized oil consumption on the liver as reflected by liver index parameters (Fig. 3). In particular, the blood TG, TC, LDL-c and HDL-c levels were returned to almost the normal status as seen in the NC group following the RS intervention (Table 1), indicating that the addition of RS could repair lipid metabolism dysfunction induced by oxidized oil. The regulation of RS supplementation on lipid metabolism might be associated with its function in improving the antioxidant status in the liver. ${ }^{37}$
Previous investigations have suggested that the alterations in lipid metabolism induced by the consumption of oxidized oils is mediated by both primary and secondary lipid peroxidation products, which are absorbed in the intestine and then transported to the liver via blood. ${ }^{38-40}$ The current study found that the functional pathways relating to adenosine monophosphate (AMP)-activated protein kinase (AMPK) were significantly enriched in the DO versus the DO-RS groups. Considering that AMPK is a major regulator of cellular energy homeostasis (glucose and lipid metabolism) in the target tissues (liver, adipose tissue, and muscle), ${ }^{41}$ these results imply that RS may be one of the key ingredients in the diet involved in the regulation of energy balance in lipid biosynthesis. The important transcription factors in hepatic lipid metabolism are peroxisome proliferator-activated receptor $\alpha(P P A R \alpha)$ and sterol regulatory element-binding protein (SREBP)-1 and -2. The results show that the consumption of oxidized oil led to the activation of PPAR $\alpha$ in the liver, which is consistent with the reports from other studies. ${ }^{\mathbf{4 2 - 4 4}}$ Furthermore, Sülzle (2004) ${ }^{\mathbf{4 5}}$ also reported the activation of the $P P A R \alpha$ pathway together with the up-regulation of PPAR $\alpha$ target genes including ACO, CYP4A1, CPT I, CPT II, medium-chain acyl-CoA dehydrogenase, long-chain acyl-CoA dehydrogenase and 3-ketoacyl-CoA dehydrogenase following the consumption of oxidized fat.

In relation to $S R E B P S$, which regulate gene expressions associated with lipid synthesis and uptake, ${ }^{\mathbf{4 6}}$ our results showed that the oxidized oil-containing diet down-regulated the expression of SREBP1 mRNA and greatly reduced the expression of lipogenesis-associated genes (Fads1, Gpam and Dgat1) in the liver. In contrast, the DO-RS diet also significantly suppressed the expression of the $S R E B P 1$ lipogenic transcription factor with an increase in lipogenesis-associated genes relative to the DO group. The role of RS in activating the AMPK pathway and its subsequent effects on SREBP activity could be associated with the production of short-chain fatty acids (SCFAs) via colonic fermentation. ${ }^{30}$ The SCFAs, mainly acetate, propionate, and butyrate stimulate colonic blood flow and fluid and electrolyte uptake, contributing to normal large bowel function and preventing pathology through their actions in the lumen and on the colonic musculature and vasculature. ${ }^{21}$ Human study also found that the concentration of SCFAs in plasma was greater following RS consumption compared to that in the control. ${ }^{47}$ Incorporation of butyrate in a high-fat diet of mice has been shown to prevent insulin resistance ${ }^{48}$ through a possible mechanism of increasing phosphorylation/activity of AMPK and p38 and subsequent expression of $P G C-1 \alpha$, resulting in mitochondrial activity and energy expenditure. Furthermore, Li et al. $(2011)^{49}$ have shown that AMPK phosphorylation of $S R E P B-1 C$ is impaired in insulin-resistant mice and that phosphorylation can occur in response to the treatment with a polyphenol, suppressing $S R E P B$ cleavage and target gene expression resulting in reduced lipogenesis and hepatic steatosis. Koch et al. $(2007)^{50}$ also found that a reduced level of cholesterol in the liver of rats following feeding with oxidized fat was mediated by an inhibition of $S R E B P-2$, which in turn led to a reduced expression of its target genes involved in hepatic cholesterol synthesis and cholesterol uptake. 
Lipogenesis (SREBP-1C), cholesterol metabolism (SREBP-2, $L X R S)$ and fatty acid oxidation (PPAR $\alpha$ ) were found to be highly associated with insulin resistance. ${ }^{50}$ In this study, we found the significant enrichment of insulin signaling pathways in the DO group versus the DO-RS group (Table 2). And the Q-PCR results also consistently showed the significant reduction in Insig-1 (although not Insig-2) expression in the DO group, which is subsequently dramatically increased in the DO-RS group, whilst SREBP1 expression was down-regulated in the DO-RS group (Table 3), indicating that RS intake partly corrects dyslipidemia and restores metabolic and inflammatory alterations in the liver induced by the oxidized oil-containing diet. This effect has also been previously reported by Polakof et al. $(2013)^{51}$ who comprehensively showed that RS could reverse insulin resistance in high-fat diet fed rats by triglyceride and cholesterol levels in the liver accompanied by the expression of SREBP$1 c$ and SREBP-2 (triglyceride and cholesterol biosynthesis respectively) and PPAR $\alpha$ (fatty acid oxidation). Insulin can regulate $S R E B P$ mediated lipogenesis through induced genes, Insig-1 and Insig-2, both of which play important roles in the regulation of intracellular cholesterol and fat metabolism ${ }^{52}$ through binding to $S R E B P-1 C$ and preventing endoplasmic reticulum to Golgi transport of SREBP-1C-SCAP complex and subsequent proteolytic cleavage and activation of SREBP-1c. Significantly decreased expression of Insig-1 and Insig-2 in the DO group is associated with a higher SREBP-1 expression, indicating the activation of lipogenesis in the liver associated with the composition of an oxidized oil diet. In contrast, the improved lipid profile in the rats of the DO-RS group was consistently accompanied by a significantly increased expression of an insulin induced gene, in particular, Insig-1. The previous report has also shown that specific hepatic overexpression of Insig-1 (or Insig-2) inhibits the activation of SREBP-1C in the liver, ${ }^{34}$ and Insig-1 has also been shown to inhibit the lipid accumulation and free fatty acid (FFA) synthesis in a time-dependent manner ${ }^{53}$ through the Insig-1SREBPS cleavage activating protein (SCAP)-SREBP-1C pathway. In this study, Insig-1 was found to be down-regulated in the rats of the DO group compared to that of the rats in the FO and NC groups, whilst after RS intervention, it was inversely regulated at the mRNA level. To further confirm the role of Insig-1, the expression of Insig-1 on protein level was revealed to be upregulated in the DO-RS group compared to that in the DO group (Fig. S2 $\dagger$ ), implying that Insig-1 is regulated at both transcriptional and post-transcriptional levels. Nevertheless, other roles of RS in the dietary cannot be ignored. For example, significantly suppressed formation of secondary bile acids and increased fecal fat excretion in RS-fed rats were also noted due to lower $\mathrm{pH}$ and higher butyrate concentration of the caecal and colonic contents induced by RS supplementation. ${ }^{54}$ Thus, a greater understanding of absorption, distribution, metabolism and excretion (ADME) properties of RS in the oxidized oilcontaining diet is also critical to fully illustrate the benefit of utilizing RS as a means to gain a better mechanism investigation. The mechanism of the impact of the oxidized oilcontaining diet with and without RS intervention on the blood lipid profile is hypothesized and presented in Fig. 5.

\section{Conclusion}

Deep-frying led to significant changes in the chemical composition of canola oil, and the lipidomic analysis revealed that the degradation and oxidation reactions occurring in the oil during deep-frying might be highly related to the increased level of DAGs and oxidized TAGs. Moreover, the lipidomic data further highlighted that unsaturated fatty acids were highly involved in these two reactions.

An oxidized oil-containing diet could induce an imbalance lipid metabolism and contribute to steatosis and liver damage in rats. The up-regulation of $P P A R \alpha$ and reduced expression of downstream lipogenesis-associated genes found to be the key factors involved in this impaired lipid metabolism in the liver. However, dietary RS intervention during the oxidized oil consumption improved the blood lipid profile through the activation of AMPK and Insig pathways with an enhanced expression of the related key genes of Prkag2 and Insig-1/2, respectively. In particular, the activation of the Insig-1/2 pathway might play the key role in improving lipid biosynthesis.

\section{Conflicts of interest}

The authors declare that there is no conflict of interest.

\section{Acknowledgements}

This study was financially supported by the National Key Research and Development Program (2016YFD0400401-2, 2016YFD0400104-4), the National Nature Science Foundation of China (No.U1501214, No. 31471701), the National Spark Program (2015GA610003), the Tianjin Research Program of Application Foundation and Advanced Technology (15JCZDJC34300) and the ARC Industrial Transformation Training Centre for Functional Grains (Australia).

\section{References}

1 I. S. Saguy and D. Dana, J. Food Eng., 2003, 56, 143-152.

2 C. Gertz, J. Lipid Sci. Technol., 2000, 102, 566-572.

3 M. A. Mendonça, W. M. Coelho Araújo, L. A. Borgo and H. C. Araújo, Journal of Culinary Science \& Technology, 2015, 13, 116-132.

4 K. Cheng, J. Shi, S. Ou, M. Wang and Y. Jiang, J. Agric. Food Chem., 2010, 58, 309-312.

5 E. Koh and J. Surh, Food Chem., 2015, 174, 467-472.

6 A. Sebastian, S. M. Ghazani and A. G. Marangoni, Food Res. Int., 2014, 64, 420-423.

7 S. Ou, Q. Lin, Y. Zhang, C. Huang, X. Sun and L. Fu, Innovative Food Sci. Emerging Technol., 2008, 9, 116-121.

8 R. Zamora and F. J. Hidalgo, J. Agric. Food Chem., 2008, 56, 6075-6080.

9 E. Capuano, T. Oliviero, Ö. Ç. Açar, V. Gökmen and V. Fogliano, Food Res. Int., 2010, 43, 1021-1026.

10 P. Lim, S. Jinap, M. Sanny, C. P. Tan and A. Khatib, J. Food Sci., 2014, 79, T115-T121.

11 J. Kanner, Mol. Nutr. Food Res., 2007, 51, 1094. 
12 K. Eder, U. Keller, F. Hirche and C. Brandsch, J. Nutr., 2003, 133, 2830-2837.

13 U. Keller, C. Brandsch and K. Eder, Eur. J. Nutr., 2004, 43, 353-359.

14 R. Ringseis, N. Piwek and K. Eder, Inflammation Res., 2007, 56, 118-125.

15 P. M. Chao, H. L. Huang, C. H. Liao, S. T. Huang and C. j. Huang, Br. J. Nutr., 2007, 98, 63-71.

16 K. Eder and G. I. Stangl, J. Nutr., 2000, 130, 116-121.

17 K. Eder, P. Skufca and C. Brandsch, J. Nutr., 2002, 132, 1275-1281.

18 P. Skufca, C. Brandsch, F. Hirche and K. Eder, Ann. Nutr. Metab., 2003, 47, 207-213.

19 R. Ringseis and K. Eder, Mol. Nutr. Food Res., 2011, 55, 109-121.

20 B. A. Ashwar, A. Gani, A. Shah, I. A. Wani and F. A. Masoodi, Starch/Staerke, 2015, 9, 555-559.

21 D. L. Topping and P. M. Clifton, Physiol. Rev., 2001, 81, 10311064.

22 Y. T. Chiu and M. L. Stewart, Asia Pac. J. Clin. Nutr., 2013, 22, 372-379.

23 M. D. Haub, K. L. Hubach, E. K. Al-tamimi, O. Sammy and P. A. Seib, J. Nutr. Metab., 2010, 230501-230504.

24 M. D. Robertson, J. M. Currie, L. M. Morgan, D. P. Jewell and K. N. Frayn, Diabetologia, 2003, 46, 659-665.

25 D. Eam and K. W. Jmm, Br. J. Nutr., 1995, 73, 287-298.

26 Y. Zhang, H. L. Zeng, Y. Wang, S. X. Zeng and B. D. Zheng, Chem. Rev., 2014, 114, 311-318.

27 X. Si, Z. K. Zhou, P. Strappe and C. Blanchard, Food Funct., 2017, 8, 232-240.

28 D. A. Kieffer, B. D. Piccolo, M. L. Marco, E. B. Kim, M. L. Goodson, M. J. Keenan, T. N. Dunn, K. E. B. Knudsen, R. J. Martin and S. H. Adams, J. Nutr., 2016, 146, 2476-2490.

29 J. Kuligowski, D. Carrión, G. Quintás, S. Garrigues and M. de la Guardia, Anal. Bioanal. Chem., 2011, 399, 1305-1314.

30 G. Shui, X. L. Guan, C. P. Low, G. H. Chua, J. S. Goh, H. Yang and M. R. Wenk, Mol. BioSyst., 2010, 6, 1008-1017.

31 K. J. Livak and T. D. Schmittgen, Methods, 2001, 25, 402-408.

32 C. Trapnell, A. Roberts, L. Goff, G. Pertea, D. Kim, D. R. Kelley, H. Pimentel, S. L. Salzberg, J. L. Rinn and L. Pachter, Nat. Protoc., 2012, 7, 562-578.

33 A. D. Attie, J. Clin. Invest., 2004, 113, 1112-1114.

34 L. J. Engelking, H. Kuriyama, R. E. Hammer, J. D. Horton, M. S. Brown, J. L. Goldstein and G. Liang, J. Clin. Invest., 2004, 113, 1168-1175.
35 X. F. Leong, M. R. Mustafa, S. Das and K. Jaarin, Lipids Health Dis., 2010, 9, 1.

36 C. Y. Ng, Y. Kamisah, O. Faizah and K. Jaarin, Int. J. Exp. Pathol., 2012, 93, 377-387.

37 N. D. Vaziri, S. M. Liu, W. L. Lau, M. Khazaeli, S. Nazertehrani, S. H. Farzaneh, D. A. Kieffer, S. H. Adams and R. J. Martin, PLoS One, 2014, 9, e114881.

38 C. Brandsch and K. Eder, Br. J. Nutr., 2004, 92, 267-275.

39 S. Luci, B. König, B. Giemsa, S. Huber, G. Hause, H. Kluge, G. I. Stangl and K. Eder, Br. J. Nutr., 2007, 97, 872-882.

40 R. Ringseis, C. Dathe, A. Muschick, C. Brandsch and K. Eder, J. Nutr., 2007, 137, 2056-2061.

41 D. G. Hardie, J. Cell Sci., 2004, 117, 5479-5487.

42 P. M. Chao, C. Y. Chao, F. J. Lin and C. j. Huang, J. Nutr., 2001, 131, 3166-3174.

43 D. Yabe, M. S. Brown and J. L. Goldstein, Proc. Natl. Acad. Sci. U. S. A., 2002, 99, 12753-12758.

44 T. Yang, P. J. Espenshade, M. E. Wright, D. Yabe, Y. Gong, R. Aebersold, J. L. Goldstein and M. S. Brown, Cell, 2002, 110, 489-500.

45 A. Sülzle, F. Hirche and K. Eder, J. Nutr., 2004, 134, 13751383.

46 M. S. Brown and J. L. Goldstein, Proc. Natl. Acad. Sci. U. S. A., 1999, 96, 11041-11048.

47 K. C. Maki, C. L. Pelkman, T. E. Finocchiaro, K. M. Kelley, A. L. Lawless, A. L. Schild and T. M. Rains, J. Nutr., 2012, 142, 717-723.

48 Z. Gao, J. Yin, J. Zhang, R. E. Ward, R. J. Martin, M. Lefevre, W. T. Cefalu and J. Ye, Diabetes, 2009, 58, 1509.

49 Y. Li, S. Xu, M. Mihaylova, B. Zheng, X. Hou, B. Jiang, O. Park, Z. Luo, E. Lefai and Y. J. Shyy, Cell Metab., 2011, 13, 376.

50 A. Koch, B. König, J. Spielmann, A. Leitner, G. I. Stangl and K. Eder, J. Nutr., 2007, 137, 2018-2023.

51 S. Polakof, M. E. Díaz-Rubio, D. Dardevet, J. F. Martin, E. Pujos-Guillot, A. Scalbert, J. L. Sebedio, A. Mazur and B. Comte, J. Nutr. Biochem., 2013, 24, 1920-1930.

52 J. L. Goldstein, R. A. DeBose-Boyd and M. S. Brown, Cell, 2006, 124, 35-46.

53 K. Chen, H. H. He, Y. H. Xie, X. Y. Xie and Z. H. Mo, J. Biomed. Sci., 2011, 18, 1.

54 M. G. Sajilata, R. S. Singhal and P. R. Kulkarni, Compr. Rev. Food Sci. Food Saf., 2006, 5, 1-17. 